\title{
The potential of using corn straw biochar to compensate the loss of available soil organic matter
}

\author{
Leisan Akhmetzyanova ${ }^{1}{ }^{,}$, Ivan Kuritsin ${ }^{2}$, Marat Zinnatov ${ }^{1}$, Alexander Gordeev ${ }^{1}$, Alina \\ Kamalova $^{1}$, and Kamalya Karamova ${ }^{1}$ \\ ${ }^{1}$ Institute of Environmental Sciences, Kazan Federal University, 420018 Kazan, Russia \\ ${ }^{2}$ LLC "Reculto", 420097 Kazan, Russia
}

\begin{abstract}
The process of migration of organic carbon from the biochar into the substrate and the aqueous phase was simulated. The process of organic carbon migration in passive and washing mode was evaluated. Part of the organic carbon was washed with water and passed into the aqueous phase, the other part remained in the mass of biochar. Biochar can be a source of available organic carbon, providing nutrition to rhizosphere microorganisms that improve the mineral nutrition of plants, both in the short and long term.
\end{abstract}

\section{Introduction}

The unreturnable removal of organic carbon from the soil with agricultural products makes the problem of soil depletion worse every year. The search and development of measures for environmentally safe farming is a key issue of modern agriculture.

Biochar has many advantages, since it improves the mechanical structure of the soil. Biochar produced from agricultural waste is also a clean and safe source of carbon. In recent years, there is a number of positive results from the introduction of biochar into the practice of agriculture in different countries [1-4]. The production of fertilizers from biochar contributes to solving the problem of storage and processing of waste from agriculture, animal husbandry, food industry and others [5]. Biochar, made on the basis of biomass, is used for sequestering carbon, sorbent for heavy metals, etc. [6, 7].

Currently, there is not enough research on the use of biochar in Russia, there is no practice of its use in agriculture. Most often, biochar is considered only as an additive that improves the physical and mechanical properties of soils, while its effect on the agrochemical properties of soils is not analyzed. There are also no exhaustive data about the biochar carbon migration process along the soil profile, and the mechanisms of interaction of biochar components with the soil. The mechanism of migration of the biochar along the soil profile needs additional research.

The aim of this work was to quantify the migration ability of the water-soluble carbon fraction of a biochar during laboratory modeling of the leaching process.

*Corresponding author: leisan-ksu@mail.ru 


\section{Materials and methods}

The object of the study is a biochar of corn straw obtained at a temperature of 400 degrees Celcius. At the first stage of the experiment, laboratory modeling of the process of passive migration of water-soluble organic carbon (WSOC) from biochar into water was performed. Dry biochar $(15 \mathrm{~g})$ was placed in conical flasks and $150 \mathrm{ml}$ of distilled water was added, stirred on a rotator for 8 hours. Then the flasks were kept in a quiet state at temperature between 21-25 degrees Celcius without exposure to direct sunlight. The exposure of the biochar in water was 3, 7, 14, 21 and 28 days. After the specified exposure periods, the determination of WSOC in an aqueous extract was carried out.

At the second stage, laboratory modeling of the process of vertical migration of WSOC of the biochar was carried out. For this 3 stainless steel box columns with a side of the walls of $30 \mathrm{~mm}$ a height of $200 \mathrm{~mm}$ were used. Aluminum oxide $\left(\mathrm{Al}_{2} \mathrm{O}_{3}\right)$ was used as a substrate in the experiment. Biochar $(50 \mathrm{~g})$ and aluminum oxide were poured into the column in a ratio of 1:3 (Fig. 1) [8,9].

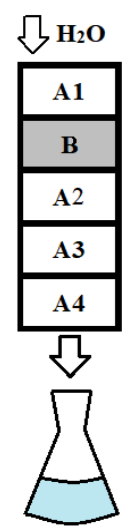

Fig. 1. The design of vertical migration columns.

The columns were irrigated from above with distilled water in $150 \mathrm{ml}$ portions. The washings were performed three times, waiting for the eluate to be completely excavated. Thus, three consecutive portions of the extract were obtained. Portions of eluate were collected in glass flasks. Aqueous extracts were examined for the content of WSOC by bichromate oxidation in a sulfuric acid medium with a spectrometric termination.

After the end of the washing, the distribution of the water-soluble carbon fraction in the migration profile was evaluated. Samples of biochar (B) and aluminum oxide (A1, A2, A3, A4) were dried to a constant mass. In the samples taken from different layers of the column after washing, the content of total, organic and inorganic carbon was determined. Determination of the total carbon content was carried out according to the Dumas method using the vario MAX cube elemental analyzer (Elementar, Germany). The content of individual carbon phases (organic and inorganic) was determined using an elemental analyzer with thermogradient analysis Leco r-612 (Leco Instruments, USA).

All parameters were measured not less than 3 times. Microsoft Excel software was used to carry out the statistical processing. The reliability of the mean differences was assessed using the Student's coefficient $(\mathrm{P}<0.05)$.

\section{Results and discussions}

On the 3 day of first stage the experiment, the concentration of WSOC in the eluate was 8.6 $\mathrm{mg} / \mathrm{ml}$ (fig.2). On the 7 day a significant increase was observed. The concentration of 
WSOC in the extract was $49 \mathrm{mg} / \mathrm{ml}$. A week later, an increase in concentration to a value of $61 \mathrm{mg} / \mathrm{ml}$ was also noted. Subsequent measurements did not show a significant change in the value of the extracted carbon during the experiment. Thus, the greatest efficiency of leaching of WSOC was observed on the 14th day of the experiment. The exposure time insignificantly changed the quantity of organic carbon extracted by water.

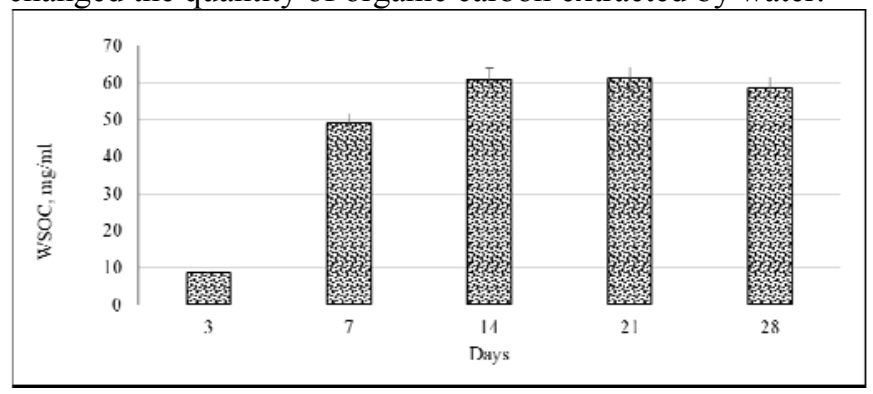

Fig. 2. The concentration of water-soluble carbon in the eluate.

The results of measurements of the concentration of organic carbon in the eluate at the second stage of the experiment are shown in Figure 3. The concentration of WSOC in the first portion of the eluate had values in the range of $7-9 \mathrm{mg} / \mathrm{ml}$. The highest concentration was observed in the second portion of the eluate and had an average value of $17.2 \mathrm{mg} / \mathrm{ml}$. The portion of eluate collected after the third washing had an average value of $7.8 \mathrm{mg} / \mathrm{ml}$.

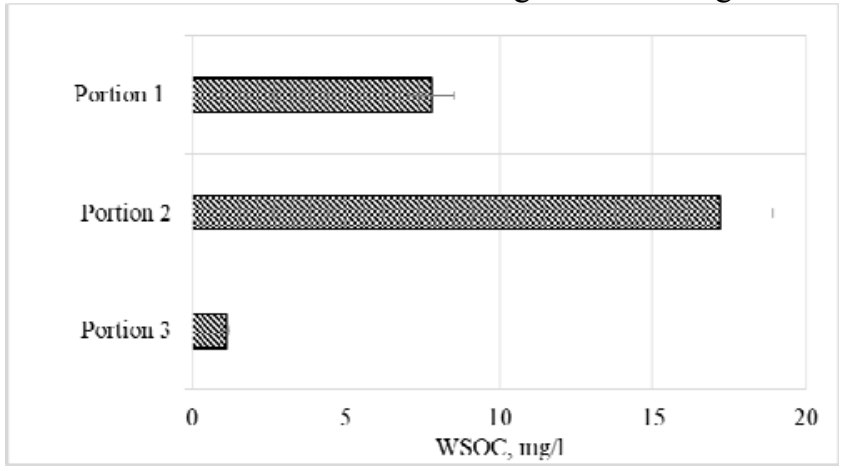

Fig. 3. The concentration of organic carbon in the eluate collected after washing the columns.

The first portions of water moisten the contents of the columns. The removal of WSOC from the mass of the biochar by subsequent portions of water becomes easier. This explains the increase in its concentration in the second portion of the eluate. Subsequent portions of water wash out the remaining amount of water-soluble compounds and in the third portion the concentration is significantly reduced. Accordingly, it can be assumed that the introduction of biochar into the substrate, as a source of organic carbon, will have greater efficiency at the initial stage, losing it during subsequent rinsing with water.

After washing the columns the carbon content in their various parts was disassembled and analyzed. The total carbon content in the biochar samples before the experiment (the initial biochar) was about $61 \%, 59 \%$ of which - organic carbon, about $2 \%$ - inorganic. Results shown in the Table 1.

Of greater interest, from the point of view of fertilizing properties, is the content of water-soluble organic carbon in different layers of the substrate profile and in the aqueous phase after washing [10]. Dissolved organic carbon is extremely important for the functioning of terrestrial ecosystems - especially for the formation of the chemical composition of soils and their genesis. [11], migration of nutrients and metals, as well as a 
substrate for soil microorganisms [12]. Due to the high content of available carbon, the nutrition of rhizosphere microorganisms that improve the mineral nutrition of plants is provided.

Table 1. Carbon content in various parts of the columns after washing.

\begin{tabular}{|c|c|c|c|}
\hline Element & Total carbon, $\mathbf{\%}_{\mathbf{w}}$ & Organic carbon, $\mathbf{\%}_{\mathbf{w}}$ & Inorganic carbon, $\mathbf{\%}_{\mathbf{w}}$ \\
\hline Initial biochar & $61,18 \pm 1,91$ & $59,185 \pm 1,85$ & $1,995 \pm 0,06$ \\
\hline A1 & $0,040 \pm 0,00166$ & $0,039 \pm 0,0016$ & $0,00158 \pm 0,00006$ \\
\hline B & $37,603 \pm 1,17$ & $36,583 \pm 1,14$ & $1,02 \pm 0,032$ \\
\hline A2 & $0,057 \pm 0,00166$ & $0,055 \pm 0,0016$ & $0,00224 \pm 0,00006$ \\
\hline A3 & $0,054 \pm 0,00166$ & $0,052 \pm 0,0016$ & $0,00194 \pm 0,00006$ \\
\hline A4 & $0,062 \pm 0,00166$ & $0,060 \pm 0,0016$ & $0,00193 \pm 0,00006$ \\
\hline
\end{tabular}

The residual amount of organic carbon in the biochar layer (B) after washing was $36.6 \%$. Thus, part of the organic carbon was washed out with water through the substrate (fig.4). The total carbon content in the aluminum oxide samples in the layer above the biochar (A1) was $0.04 \%$, the largest share in this case was organic carbon $(0.039 \%)$. The content of organic carbon in the layers of aluminum oxide under the biochar is 1.4 times higher than in the upper one. Consequently, this component of biochar migrates down the profile, and lingers in the substrate, albeit in small quantities.

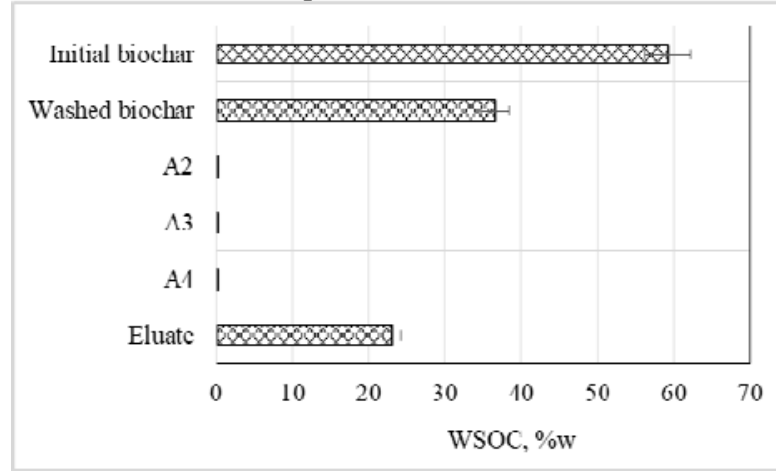

Fig. 4. The concentration of organic carbon in different layers of columns.

A small amount of carbon has been fixed on the substrate, under natural conditions, an increase in this value should be expected [13]. Organic matter from the biochar in the soil will be retained not only mechanically, but also at the level of chemical interaction with soil organic matter determined by its content and structure. The method of agricultural tillage, soil structure and filtration coefficient also are important.

The loss of organic carbon from the mass of the biochar after washing are about $23 \%$. Consequently, corn straw biochar consists of almost a quarter of WSOC and can act as a source of available organic carbon. Which, under conditions of sufficient moisture, can become an additional source of nutrients for soil microorganisms. A. Obia found out that 
$19 \%$ of carbon from biochar migrated to the lower layers of the soil. The penetration depth is $30-40 \mathrm{~cm}$ from the biochar layer and increases over time [12].

It is shown that the components of the biochar are capable of vertical migration along the soil profile $(30-50 \mathrm{~cm})$. The authors attribute this to direct leaching and infiltration of biochar in soluble or solid forms due to a large amount of precipitation, or ingestion and bio-displacement by earthworms [11].

Analyzing the data obtained, it can be assumed that the corn straw biochar consists of $59 \%$ organic carbon. On the one hand, it can serve as a fast source of organic matter ( $23 \%$ WSOC). On the other hand, part of the organic carbon remains in the mass of the biochar and has the potential, due to the activity of living organisms, to slowly pass into the substrate and further into the soil solution, thereby enriching it with available organic matter $[14,15]$. Thus, the use of corn straw biochar can have a beneficial effect on the nutrition conditions of rhizosphere microorganisms and, as a consequence, plants by improving the properties of the soil substrate.

\section{Conclusion}

The main part of the carbon of the biochar from corn straw belongs to the total organic carbon, the proportion of inorganic carbon in the samples is insignificant.

Passive exposure of the biochar in water showed the greatest efficiency of leaching of WSOC from biochar was observed on the 14th day of the experiment. The exposure time insignificantly changed the quantity of organic carbon extracted by water.

The water-soluble organic carbon of the corn straw biochar is able to migrate quite actively along the vertical profile into the aqueous extract. Carbon capture on an inert substrate is insignificantly.

Biochar produced from agricultural waste can be a clean, safe source of available organic carbon and can have a beneficial effect on the soil biota.

\section{References}

1. J. Lehmann, S. Biol. Bioch., 43, 1812-1836 (2011)

2. S. Jeffery, Agr. Ecosyst. Env., 144,175-187 (2011)

3. D.N. Mulcahy, J. Arid. Env., 88, 222-225 (2013)

4. P. Blackwell, G. P. Riethmuller, Earthscan, (2009)

5. S.P. Galinato, J.K. Yoder, D. Granatstein, Enrg. Pol., 10, 6344-6350 (2011)

6. K. Weber, P. Quicker, Fuel. Elsevier., 217, 240-261 (2018)

7. P. Galitskaya, L. Akhmetzyanova, S. Selivanovskaya, Biogeosci., 13, 5739-5752 (2016)

8. A. Giniyatullin, Res. J. Pharm. Biol. Chem. Sci., 4, 194-201 (2015)

9. McKnight D.M., Env. Sci. Tech., 7, 1388-1396 (1992)

10. F. Santos, S. Biol. Bioch., 51, 115-124 (2012)

11. Y. Fang, Plos One, 43, 48 (2015)

12. A. Obia, S. Till. Res., 40, 30-34 (2017)

13. A. Keith, Env. Sci. Tech. 45, 9611-9618 (2011)

14. Y. Kuzyakov, S. Biol. Bioch., 32, 1485-1491 (2000)

15. I. J. Umaru, B. Samling, U.H. Aduwamai, As. J. Bioch. Gen. Mol. Biol., 10, 12-15 (2019) 